\title{
Inhibition of Systemic Hyaluronan Synthesis Exacerbates Murine Hepatic Carcinogenesis
}

\author{
KENICHIRO MIKAMI, TETSU ENDO, NAOYA SAWADA, GO IGARASHI, \\ MASAYO KIMURA, HIROTAKE SAKURABA and SHINSAKU FUKUDA \\ Department of Gastroenterology and Hematology, \\ Hirosaki University Graduate School of Medicine, Hirosaki, Japan
}

\begin{abstract}
Background/Aim: Hyaluronan (HA) is used as a biomarker of liver fibrosis, which is a key risk factor for the development of hepatocellular carcinoma (HCC). We examined the effects of prolonged pharmacological inhibition of HA synthesis on liver carcinogenesis. Materials and Methods: Liver tumors were induced in mice by administering $0.03 \%$ thioacetamide (TAA) in drinking water over a 12-month period. Animals simultaneously received either a diet containing of an inhibitor of HA synthesis [4methylumbelliferone (4-MU)], or a control diet. Results: Addition of 4-MU resulted in a significantly higher number of tumors compared to TAA treatment alone. Moreover, addition of 4-MU resulted in a dose-dependent increase in maximum tumor size. Conclusion: While local HA suppression has been shown to have an inhibitory effect on $H C C$ in vitro and in tumor cell implantation experiments, the present results indicate that systemic inhibition of $H A$ synthesis by 4-MU supplementation facilitates hepatic carcinogenesis in vivo.
\end{abstract}

Hepatocellular carcinoma (HCC) is the sixth most common cancer and the second leading cause of cancer-related deaths in the world (1). It is estimated that the incidence and mortality of HCC are increasing steadily (2). Therefore, $\mathrm{HCC}$ will continue to be a serious social concern for several decades, and it is critical to develop an effective anticancer therapy. The relationship between chronic liver disease,

This article is freely accessible online.

Correspondence to: Kenichiro Mikami, Department of Gastroenterology and Hematology, Hirosaki University Graduate School of Medicine, 5 Zaifu-cho, Hirosaki, Aomori 036-8562, Japan. Tel: +81 172395053, Fax: +81 172375946, e-mail: kmikami@ hirosaki-u.ac.jp

Key Words: Hepatocellular carcinoma, hyaluronan, 4-methylumelliferone. including cirrhosis, and HCC is clear. Most HCC cases arise in the setting of chronic liver disease or cirrhosis due to chronic viral infection, excessive alcohol intake, and nonalcoholic fatty liver disease (3). In chronic liver disease, the extracellular matrix (ECM) including hyaluronan (HA) and collagen deposits in the liver depend on fibrosis progression, regardless of the etiology (4). In fact, elevation of serum levels of HA and collagen has been found in patients with chronic liver disease, and these can be used as a noninvasive biomarker to assess the progression of liver fibrosis (5).

$\mathrm{HA}$ is an anionic glycosaminoglycan consisting of repeating polymeric disaccharides $\mathrm{N}$-acetylglucosamine and glucuronic acid. It is a major component of the ECM and exists ubiquitously in human tissues (6). HA has various functions in a normal biological state, such as hydration, lubrication of joints, space filling, and provision of a matrix through which cells can migrate (7). During tissue injury, HA is actively produced for tissue repair with regulation of epithelial cell and fibroblast behavior $(8,9)$. In regard to cancer, many previous studies have demonstrated that stromal HA may create a permissive extracellular microenvironment for tumor progression and metastasis through cancer cell proliferation, migration, and invasion (10-16). An increase in HA deposits has been correlated with poor clinical prognosis in pancreatic, colorectal, ovarian, and breast cancer (17). Thus, HA signaling is expected to be a target for anticancer therapy (18).

The coumarin derivative 4-methylumbelliferone (4-MU) inhibits HA production via depletion of cellular uridine diphosphate glucuronic acid and down-regulation of expression of HA synthase 2 and $3(19,20)$. 4-MU has been clinically used for the treatment of functional and obstructive biliary tract spasms, but recent experimental studies have demonstrated that 4-MU may have other potential therapeutic benefits for treating cancer in various organs such as the pancreas, prostate, skin, breast, and ovaries (21-25). 4-MU also has antitumor effects in HCC that are similar in other cancer types with in vitro and tumor cell transplantation models $(26,27)$. However, the therapeutic effects of 4-MU on liver carcinogenesis have not 
yet been studied. Of particular note is the pathogenesis of HCC that characteristically develops based on chronic liver damage during the course of progression of fibrosis in the liver $(3,4)$. For this purpose, it is important to clarify the effects of 4-MU on liver carcinogenesis.

In this study, we investigated whether 4-MU supplementation would inhibit murine liver carcinogenesis induced by administration of thioacetamide (TAA), which causes chronic liver damage and fibrosis, as previous studies showed the beneficial effect of 4-MU in in vitro and tumor cell implantation models $(26,27)$.

\section{Materials and Methods}

Materials. TAA and 4-MU were purchased from Wako Pure Chemical Industries, Ltd. (Osaka, Japan) and Sigma-Aldrich (St. Louis, MO, USA), respectively. Chow containing 4-MU was pelleted by Oriental Yeast Co., Ltd. (Tokyo, Japan).

Animals and treatments. All animals received humane care, with the study being conducted in accordance with Hirosaki University's Guidelines for Animal Experimentation. C57B1/6J mice were obtained from CLEA Japan (Tokyo, Japan). Animals were housed in cages in a temperature- and humidity-controlled room with a 12-hour light/dark cycle, and they were given free access to food and water.

Following acclimation, 5-week-old male C57B1/6J mice received TAA at a concentration of $0.03 \%$ in drinking water to induce hepatic tumorigenesis. The control mice were fed a standard diet without TAA administration, whereas TAA-treated mice were simultaneously fed either a diet containing 4-MU $(0.01,0.1,1.0$, or $5.0 \%$; $n=4-$ 5/group) or a standard diet. After 12 months, the mice were sacrificed. Liver, spleen, and blood samples were collected for analyses. Liver body weight index and spleen weight index were calculated as liver weight and spleen weight in proportion to body weight, respectively.

Serum biochemical analyses. Serum alanine transaminase (ALT), alkaline phosphatase (ALP), lactate dehydrogenase (LDH), and total bilirubin (T-bil) were measured with an automated analyzer (Spotchem EZ SP-4430; Arkrey Inc., Kyoto, Japan).

Liver tumor analysis. The number of visible tumor nodules on the liver surface was counted macroscopically. The maximum tumor size was determined by measuring the diameter of the largest tumor nodule on the liver surface for each liver.

Statistical analysis. Quantitative values are expressed as the mean \pm standard error of the mean (SEM). Statistical evaluations were performed using two-tailed Student's $t$-test. Differences were considered to be significant with $p$-values of less than 0.05 .

\section{Results}

Survival and weight measurements. While lower 4-MU concentrations (0.01-1.0\%) did not affect survival, all mice that received TAA died within one week with addition of 5\% 4-MU. Significantly lower body weight was seen in TAA-treated mice, while the addition of 4-MU did not significantly affect body

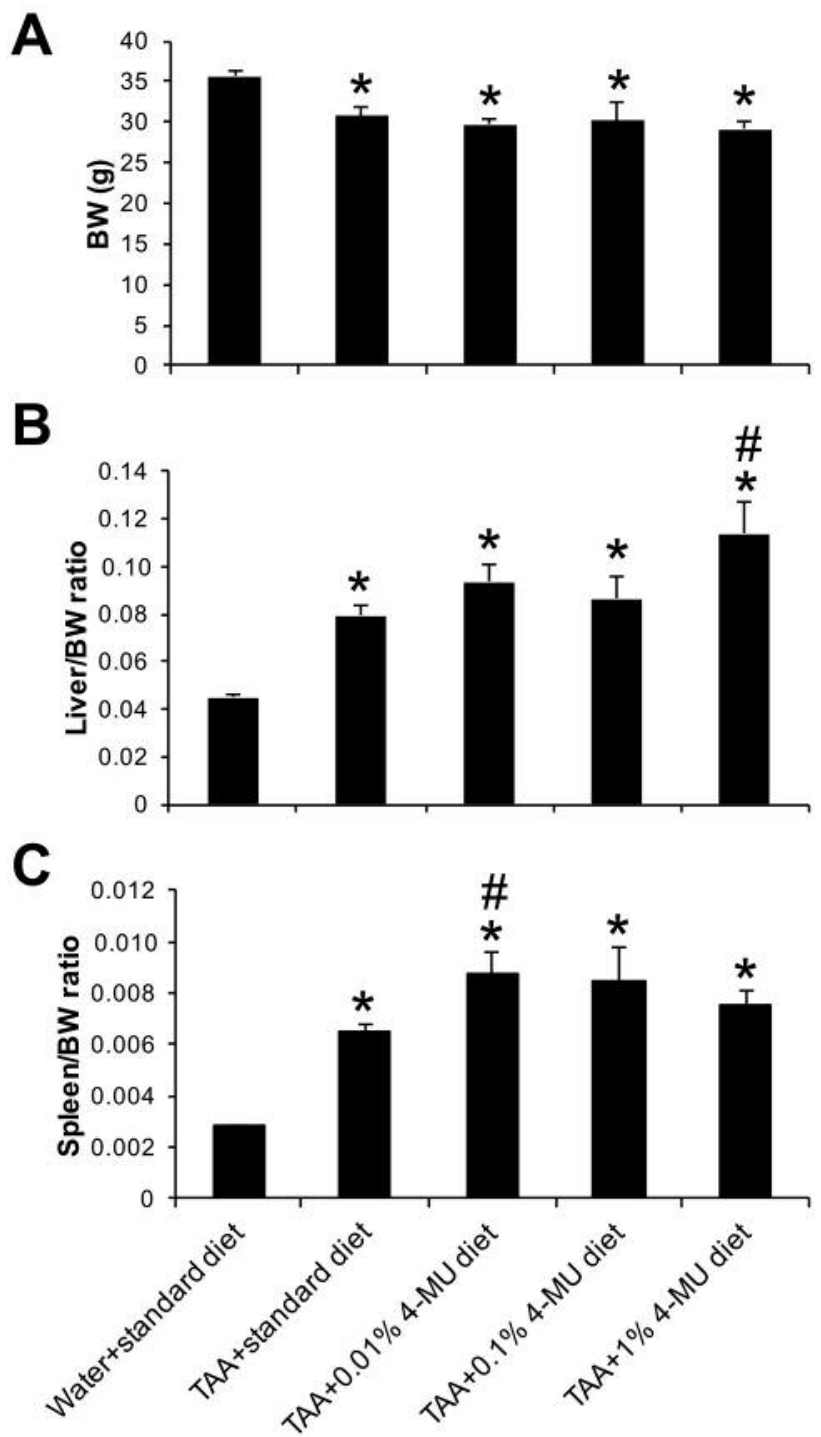

Figure 1. Systemic inhibition of hyaluronan (HA) synthesis induces hepatomegaly and splenomegaly in mice treated with the hepatic carcinogenic thioacetamide (TAA). Body weight $(B W)(A)$, liver weight to body weight ratio $(B)$, and spleen weight to body weight ratio $(C)$ were determined in mice treated with TAA for 12 months with or without 4-MU supplementation. Student's t-test: significantly different at $p<0.05$ compared to ${ }^{*}$ water + standard diet, and ${ }^{\#}$ TAA + standard diet, $n=4-5$.

weight (Figure 1A). TAA treatment induced hepatomegaly (increased liver weight to body weight ratio) and splenomegaly (increased spleen weight to body weight ratio), and both were exacerbated by addition of 4-MU (Figure 1B and C).

Biochemistry. 4-MU supplementation induced significant exacerbation of liver injury in TAA-treated mice, as indicated by an increase in serum ALT, ALP, and LDH levels (Figure 2A-C). No significant change was observed in serum 
A

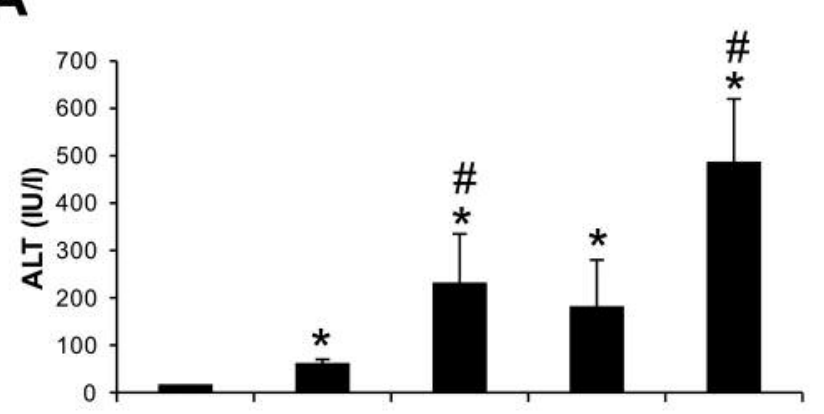

C
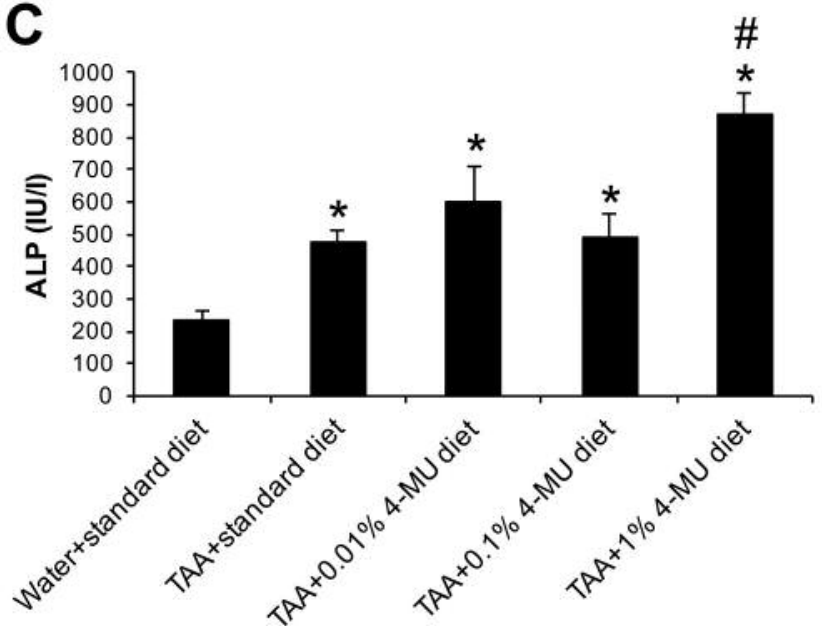

B

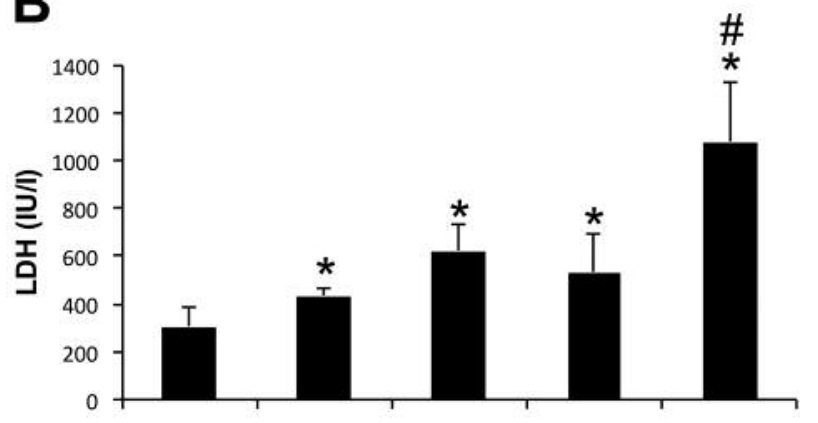

D

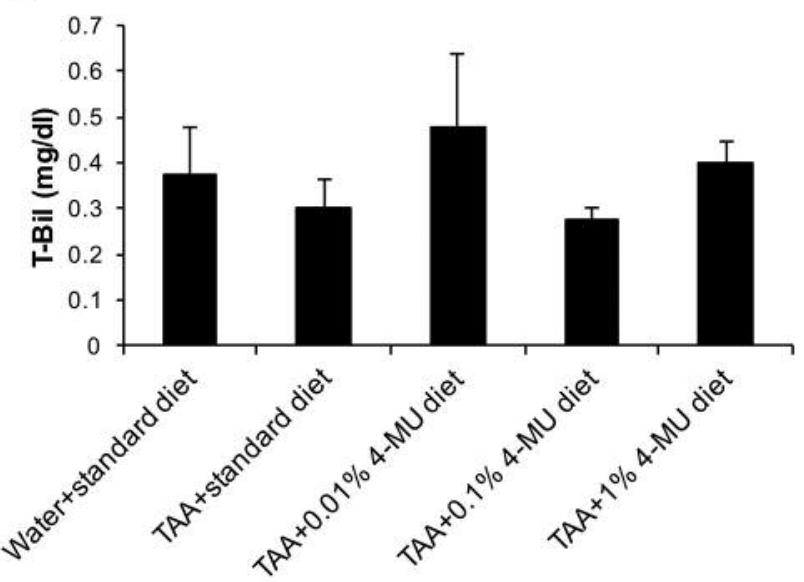

Figure 2. Systemic inhibition of hyaluronan (HA) synthesis worsens liver injury in mice treated with the hepatic carcinogenic thioacetamide (TAA). Serum alanine transaminase (ALT) (A), lactate dehydrogenase $(L D H)(B)$, alkaline phosphatase $(A L P)(C)$ and total bilirubin $(T-B i l)(D)$ levels were measured in mice treated with TAA for 12 months with or without 4-MU supplementation. Student t-test: significantly different at p<0.05 compared to ${ }^{*}$ water + standard diet, and ${ }^{\#} T A A+$ standard diet, $n=4-5$.

T-Bil level in TAA-treated mice with or without 4-MU supplementation (Figure 2D).

Tumor measurement. Administration of TAA induced liver tumors (TAA alone, number of tumors: $6.2 \pm 1.5$ ), while mice that did not receive TAA had no tumors. It is important to note that the number of tumors increased significantly with the addition of 4-MU compared to mice that received TAA alone (TAA+0.01\% 4-MU: $19.0 \pm 3.5$; TAA+0.1\% 4-MU: 10.5 $\pm 4.0 ;$ TAA $+1 \%$ 4-MU: $22.2 \pm 6.5 ; p<0.05)$. Moreover, a dose-dependent increase in maximum tumor size was found with addition of 4-MU. Taken together, these findings indicate that systemic inhibition of HA exacerbates hepatic carcinogenesis in TAA-treated mice.

\section{Discussion}

Here, we showed a HCC development in a hepatic carcinogenic mouse model. Previous reports have shown the antitumor effectiveness of HA inhibition by 4-MU in HCC $(20,21)$. However, those studies were conducted with an in vitro or tumor cell implantation model. In the present study, however, we tested an experimental HCC carcinogenesis model accompanied by chronic liver damage induced by TAA to mimic human hepatic carcinogenesis. The reason is that HCC clearly develops based on chronic liver injury due to causes such as hepatitis virus infection, alcohol, and nonalcoholic steatohepatitis (3).

The tumor microenvironment plays a critical role in cancer development and progression with the interaction between tumor cells and ECM molecules (28). As an integral component of the ECM, HA influences the behavior of tumor and stromal cells in proliferation, motility, invasion, and stemness through the activation of phosphatidylinositol3 kinase (PI3K)/AKT and extracellular signal-regulated kinase $1 / 2$ (ERK1/2) signaling pathways (29, 30). HA accumulation in tumor tissues indicates tumor progression and poor prognosis in patients with cancer (31-34). Thus, 
A

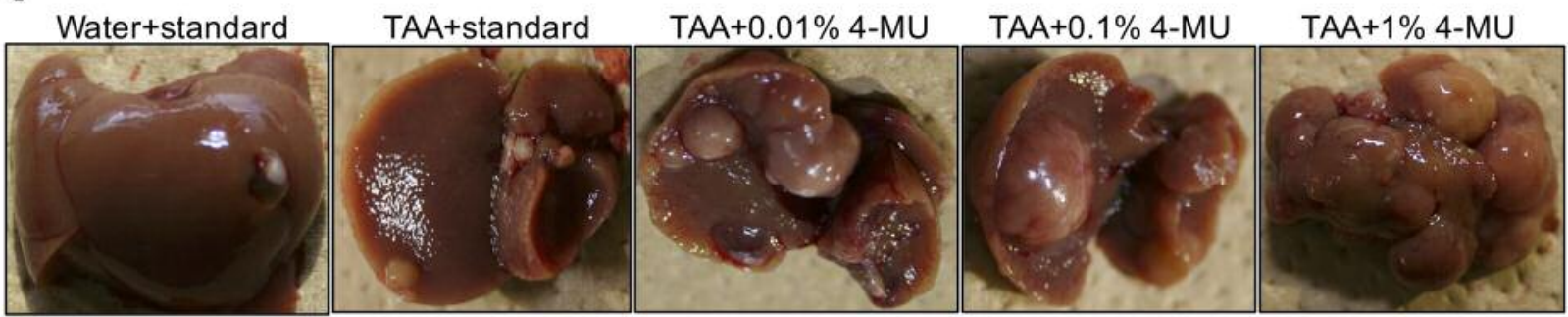

B

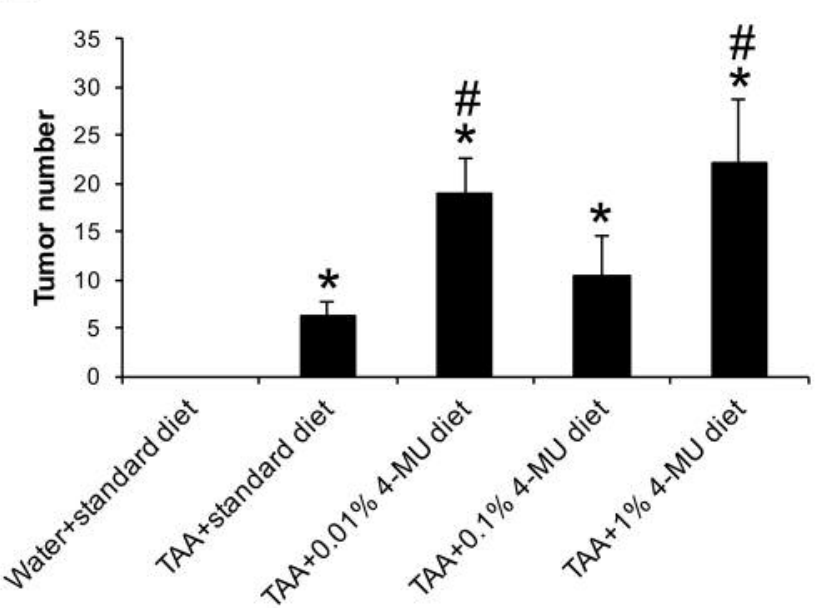

C

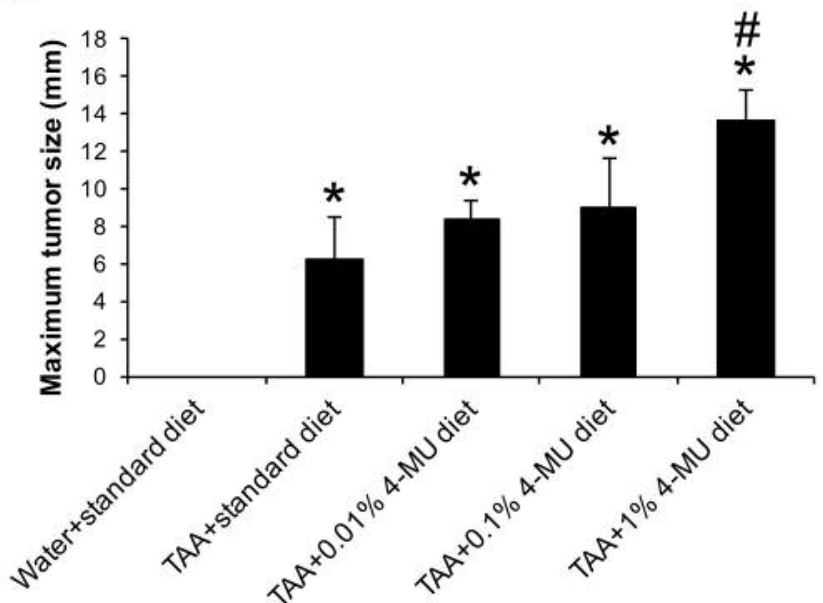

Figure 3. Systemic inhibition of hyaluronan (HA) synthesis aggravates liver tumor progression in mice treated with the hepatic carcinogenic thioacetamide (TAA). A: Representative macroscopic images of livers in mice treated with TAA for 12 months with or without 4-MU supplementation. Number of tumors $(B)$ and maximum tumor size $(C)$ in liver from indicated groups. Student's $t$-test: significantly different at $p<0.05$ compared to *water + standard diet, and ${ }^{\#} T A A+$ standard diet, $n=4-5$.

many researchers have focused on HA signaling as a target for cancer therapy.

Many recent experimental studies of 4-MU have shown it to have antitumor activity against various types of cancer by inhibiting HA synthesis (21-25). Almost all of those studies were implemented in cell culture or an implantation model of a cancer cell line. HA modulates tumor cell behaviors such as proliferation, motility, and metastatic spread through the tumor microenvironment. Therefore, it is speculated that the results of these experiments with 4-MU in various cancer cells show its effectiveness in terms of antitumor activity through the tumor microenvironment. However, in the process of carcinogenesis, there are many factors that affect the development of cancer, not only those in the local tumor microenvironment but also systemic factors such as the immune system, cytokines, and nutrition (35-37).

Furthermore, HCC develops based on chronic liver disease, which involves progression of liver fibrosis and cirrhosis, with accumulation of ECM including HA (4).
Therefore, it is expected that the development of HCC is closely related to HA signaling. In fact, a high preoperative serum HA level was found to correlate with a poor prognosis in patients with HCC (31). Indeed, overexpression of HA promoted progression of HCC cell lines (38). Conversely, suppression of HA expression by using 4-MU inhibited progression of HCC cell lines $(26,27)$. These previous reports were conducted using in vitro and tumor cell implantation models with HCC cell lines. To broaden the scope to translational research, we examined the effect of systemic inhibition of HA synthesis in a TAA-induced hepatic carcinogenesis model. Contrary to our expectations, however, our results showed that the systemic inhibition of HA synthesis actually promoted the development of liver tumors. Although the precise mechanisms for why the systemic inhibition of HA synthesis promoted HCC are unclear, considering both the previous reports and our results, the local inhibition of HA synthesis in the tumor microenvironment undoubtedly is a critical factor for the 
decline of HCC. In order to develop a new therapeutic approach for HCC with regulation of HA signaling, further studies are needed to clarify the effects of HA inhibition not only in the liver but also in the whole body.

In conclusion, we found that systemic inhibition of HA synthesis by oral 4-MU administration promoted the development of liver tumors in TAA-treated mice. Despite the fact that many studies have shown that the inhibition of HA synthesis by 4-MU suppresses the progression of cancer in various organs, including the liver, our findings showed the completely opposite effect in liver tumors. In light of the focus on development of a new cancer therapy with regulation of HA signaling, our findings suggest that further studies are needed to reveal the effects of HA inhibition not only in the tumor microenvironment but also in systemic reactions. On the other hand, since local inhibition of HA synthesis is obviously effective in various cancer cell lines including $\mathrm{HCC}$, it is also important to explore new procedures to control the local HA signaling in the tumor microenvironment. Regulation of HA signaling is a promising strategy for cancer therapy; thus, further studies are required to establish an effective therapeutic approach against $\mathrm{HCC}$.

\section{Acknowledgements}

The Authors thank Dr. Ikuko Kakizaki for constructive advice. This work was supported by intramural funding received from Hirosaki University Graduate School of Medicine.

\section{Disclosure}

The Authors who took part in this study declare that they do not have anything to disclose regarding funding or conflicts of interest with respect to this article.

\section{References}

1 Torre LA, Bray F, Siegel RL, Ferlay J, Lortet-Tieulent J and Jemal A: Global cancer statistics, 2012. CA Cancer J Clin 65: 87-108, 2015.

2 Choo SP, Tan WL, Goh BK, Tai WM and Zhu AX: Comparison of hepatocellular carcinoma in Eastern versus Western populations. Cancer 122: 3430-3446, 2016.

3 Fattovich G, Stroffolini T, Zagni I and Donato F: Hepatocellular carcinoma in cirrhosis: incidence and risk factors. Gastroenterology 127: S35-50, 2004.

4 Wallace MC and Friedman SL: Hepatic fibrosis and the microenvironment: fertile soil for hepatocellular carcinoma development. Gene Expr 16: 77-84, 2014.

5 Neuman MG, Cohen LB and Nanau RM: Hyaluronic acid as a non-invasive biomarker of liver fibrosis. Clin Biochem 49: 302$315,2016$.

6 Spicer AP and McDonald JA: Characterization and molecular evolution of a vertebrate hyaluronan synthase gene family. J Biol Chem 273: 1923-1932, 1998.
7 Toole BP: Hyaluronan: from extracellular glue to pericellular cue. Nat Rev Cancer 4: 528-539, 2004.

8 Slevin M, Krupinski J, Gaffney J, Matou S, West D, Delisser H, Savani RC and Kumar S: Hyaluronan-mediated angiogenesis in vascular disease: uncovering RHAMM and CD44 receptor signaling pathways. Matrix Biol 26: 58-68, 2007.

9 Jiang D, Liang J, Fan J, Yu S, Chen S, Luo Y, Prestwich GD, Mascarenhas MM, Garg HG, Quinn DA, Homer RJ, Goldstein DR, Bucala R, Lee PJ, Medzhitov R and Noble PW: Regulation of lung injury and repair by Toll-like receptors and hyaluronan. Nat Med 11: 1173-1179, 2005.

10 Chanmee T, Ontong P and Itano N: Hyaluronan: A modulator of the tumor microenvironment. Cancer Lett 375: 20-30, 2016.

11 Schmaus A, Klusmeier S, Rothley M, Dimmler A, Sipos B, Faller G, Thiele W, Allgayer H, Hohenberger P, Post S and Sleeman JP: Accumulation of small hyaluronan oligosaccharides in tumour interstitial fluid correlates with lymphatic invasion and lymph node metastasis. Br J Cancer 111: 559-567, 2014.

12 Bernert B, Porsch $\mathrm{H}$ and Heldin P: Hyaluronan synthase 2 (HAS2) promotes breast cancer cell invasion by suppression of tissue metalloproteinase inhibitor 1 (TIMP-1). J Biol Chem 286: 42349-42359, 2011.

13 Kobayashi N, Miyoshi S, Mikami T, Koyama H, Kitazawa M, Takeoka M, Sano K, Amano J, Isogai Z, Niida S, Oguri K, Okayama M, McDonald JA, Kimata K, Taniguchi S and Itano $\mathrm{N}$ : Hyaluronan deficiency in tumor stroma impairs macrophage trafficking and tumor neovascularization. Cancer Res 70: 70737083, 2010 .

14 Koyama H, Hibi T, Isogai Z, Yoneda M, Fujimori M, Amano J, Kawakubo M, Kannagi R, Kimata K, Taniguchi S and Itano N: Hyperproduction of hyaluronan in neu-induced mammary tumor accelerates angiogenesis through stromal cell recruitment: possible involvement of versican/PG-M. Am J Pathol 170: 10861099, 2007.

15 Sugahara KN, Murai T, Nishinakamura H, Kawashima H, Saya $\mathrm{H}$ and Miyasaka M: Hyaluronan oligosaccharides induce CD44 cleavage and promote cell migration in CD44-expressing tumor cells. J Biol Chem 278: 32259-32265, 2003.

16 Itano N, Sawai T, Miyaishi O and Kimata K: Relationship between hyaluronan production and metastatic potential of mouse mammary carcinoma cells. Cancer Res 59: 2499-2504, 1999.

17 Tammi RH, Kultti A, Kosma VM, Pirinen R, Auvinen P and Tammi MI: Hyaluronan in human tumors: pathobiological and prognostic messages from cell-associated and stromal hyaluronan. Semin Cancer Biol 18: 288-295, 2008.

18 Karousou E, Misra S, Ghatak S, Dobra K, Götte M, Vigetti D, Passi A, Karamanos NK and Skandalis SS: Roles and targeting of the HAS/hyaluronan/CD44 molecular system in cancer. Matrix Biol 59: 3-22, 2017.

19 Kakizaki I, Kojima K, Takagaki K, Endo M, Kannagi R, Ito M, Maruo Y, Sato H, Yasuda T, Mita S, Kimata K and Itano N: A novel mechanism for the inhibition of hyaluronan biosynthesis by 4-methylumbelliferone. J Biol Chem 279: 33281-33289, 2004.

20 Kultti A, Pasonen-Seppänen S, Jauhiainen M, Rilla KJ, Kärnä R, Pyöriä E, Tammi RH and Tammi MI: 4-Methylumbelliferone inhibits hyaluronan synthesis by depletion of cellular UDPglucuronic acid and downregulation of hyaluronan synthase 2 and 3. Exp Cell Res 315: 1914-1923, 2009. 
21 Nagase H, Kudo D, Suto A, Yoshida E, Suto S, Negishi M, Kakizaki I and Hakamada K: 4-Methylumbelliferone suppresses hyaluronan synthesis and tumor progression in SCID mice intraabdominally inoculated with pancreatic cancer cells. Pancreas 46: 190-197, 2017.

22 Lokeshwar VB, Lopez LE, Munoz D, Chi A, Shirodkar SP, Lokeshwar SD, Escudero DO, Dhir N and Altman N: Antitumor activity of hyaluronic acid synthesis inhibitor 4-methylumbelliferone in prostate cancer cells. Cancer Res 70: 2613-2623, 2010.

23 Edward M, Quinn JA, Pasonen-Seppänen SM, McCann BA and Tammi RH: 4-Methylumbelliferone inhibits tumour cell growth and the activation of stromal hyaluronan synthesis by melanoma cell-derived factors. Br J Dermatol 162: 1224-1232, 2010.

24 Urakawa H, Nishida Y, Wasa J, Arai E, Zhuo L, Kimata K, Kozawa E, Futamura $\mathrm{N}$ and Ishiguro $\mathrm{N}$ : Inhibition of hyaluronan synthesis in breast cancer cells by 4-methylumbelliferone suppresses tumorigenicity in vitro and metastatic lesions of bone in vivo. Int J Cancer 130: 454-466, 2012.

25 Tamura R, Yokoyama Y, Yoshida H, Imaizumi T and Mizunuma $\mathrm{H}$ : 4-Methylumbelliferone inhibits ovarian cancer growth by suppressing thymidine phosphorylase expression. J Ovarian Res 7: 94, 2014

26 Piccioni F, Malvicini M, Garcia MG, Rodriguez A, Atorrasagasti C, Kippes N, Piedra Buena IT, Rizzo MM, Bayo J, Aquino J, Viola M, Passi A, Alaniz L and Mazzolini G: Antitumor effects of hyaluronic acid inhibitor 4-methylumbelliferone in an orthotopic hepatocellular carcinoma model in mice. Glycobiology 22: 400-410, 2012.

27 Piccioni F, Fiore E, Bayo J, Atorrasagasti C, Peixoto E, Rizzo M, Malvicini M, Tirado-González I, García MG, Alaniz L and Mazzolini G: 4-methylumbelliferone inhibits hepatocellular carcinoma growth by decreasing IL-6 production and angiogenesis. Glycobiology 25: 825-835, 2015.

28 Bissell MJ, Radisky DC, Rizki A, Weaver VM and Petersen OW: The organizing principle: microenvironmental influences in the normal and malignant breast. Differentiation 70: 537-546, 2002.

29 Zhu R, Wang SC, Sun C, Tao Y, Piao HL, Wang XQ, Du MR and Da-Jin Li: Hyaluronan-CD44 interaction promotes growth of decidual stromal cells in human first-trimester pregnancy. PLoS One 8: e74812, 2013.
30 Lokeshwar VB, Mirza S and Jordan A: Targeting hyaluronic acid family for cancer chemoprevention and therapy. Adv Cancer Res 123: 35-65, 2014.

31 Wu RL, Huang L, Zhao HC and Geng XP: Hyaluronic acid in digestive cancers. J Cancer Res Clin Oncol 143: 1-16, 2017.

32 Wang D, Narula N, Azzopardi S, Smith RS, Nasar A, Altorki NK, Mittal V, Somwar R, Stiles BM and Du YN: Expression of the receptor for hyaluronic acid-mediated motility (RHAMM) is associated with poor prognosis and metastasis in non-small cell lung carcinoma. Oncotarget 7: 39957-39969, 2016.

33 Peng C, Wallwiener M, Rudolph A, Ćuk K, Eilber U, Celik M, Modugno C, Trumpp A, Heil J, Marmé F, Madhavan D, Nees J, Riethdorf S, Schott S, Sohn C, Pantel K, Schneeweiss A, ChangClaude J, Yang R and Burwinkel B: Plasma hyaluronic acid level as a prognostic and monitoring marker of metastatic breast cancer. Int J Cancer 138: 2499-2509, 2016.

34 Kramer MW, Escudero DO, Lokeshwar SD, Golshani R, Ekwenna OO, Acosta K, Merseburger AS, Soloway M and Lokeshwar VB: Association of hyaluronic acid family members (HAS1, HAS2, and HYAL-1) with bladder cancer diagnosis and prognosis. Cancer 117: 1197-1209, 2011.

35 Sangaletti S, Chiodoni C, Tripodo C and Colombo MP: Common extracellular matrix regulation of myeloid cell activity in the bone marrow and tumor microenvironments. Cancer Immunol Immunother 66: 1059-1067, 2017.

36 Macintyre AN and Rathmell JC: Activated lymphocytes as a metabolic model for carcinogenesis. Cancer Metab 1: 5, 2013.

37 Valdés-Ramos R and Benítez-Arciniega AD: Nutrition and immunity in cancer. Br J Nutr 98: S127-132, 2007.

$38 \mathrm{Li} \mathrm{JH}$, Wang YC, Qin CD, Yao RR, Zhang R, Wang Y, Xie XY, Zhang L, Wang YH and Ren ZG: Over expression of hyaluronan promotes progression of HCC via CD44-mediated pyruvate kinase M2 nuclear translocation. Am J Cancer Res 6: 509-521, 2016. 